\title{
First Test of a Partial Siberian Snake for Acceleration of Polarized Protons
}

\author{
D.D. Caussyn*, R. Baiod ${ }^{\ddagger}$, B.B. Blinov*,(a), C.M. Chu*, E.D. Courant,(b) \\ D.A. Crandell ${ }^{*}$, Ya.S. Derbenev ${ }^{\dagger,(c)}$, T.J.P. Ellison ${ }^{\dagger}$, W.A. Kaufman ${ }^{*}$, \\ A.D. Krisch*, S.Y. Lee ${ }^{\dagger}$, M.G. Minty ${ }^{\dagger \dagger}$, T.S. Nurushev*, C. Ohmori ${ }^{\natural}$, \\ R.A. Phelps*, D.B. Raczkowski*, L.G. Ratner*,(b), P. Schwandt ${ }^{\dagger}$, \\ E.J. Stephenson ${ }^{\dagger}$, F. Sperisen ${ }^{\dagger}$, B. von Przewoski ${ }^{\dagger}$, U. Wienands ${ }^{\prime l}($ (d), \\ V.K. Wong*,(e) \\ "Randall Laboratory of Physics, University of Michigan, Ann Arbor, Michigan $48109-1120$ \\ 'Indiana University Cyclotron Facility, Bloomington, Indiana 47408-0768 \\ 'Fermilab, Batavia, Illinois 60510 \\ ${ }^{\$}$ Institute for Nuclear Study, University of Tokyo, Tanashi, Tokyo 188, Japan \\ ${ }^{\dagger}$ Stanford Linear Accelerator Center, Stanford, California 94309 \\ "SSC Lab, Dallas, Texae
}

\begin{abstract}
We recently studied the first acceleration of a spin-polarised proton beam through a depolarising resonance using a partial Siberian snake. We accelerated polarired protons from 95 to $140 \mathrm{MeV}$ with a constant $10 \%$ partial Siberian snake obtained using rampable solenoids. The $10 \%$ partial snake suppressed all observable depolarization during acceleration due to the $G \gamma=2$ imperfection depolarizing resonance which occurred near $108 \mathrm{MeV}$. However, $20 \%$ and $30 \%$ partial Siberian snakes apparently moved an intrinsic depolarizing resonance, normally near $177 \mathrm{MeV}$, into our energy range; this caused some interesting, although not-yet-fully understood, depolarization.
\end{abstract}

\section{INTRODUCTION}

Many depolarizing resonances are encountered in accelerating a polarized proton beam to high energies in circular accelerators. Polarized proton beams have been accelerated by correcting each individual resonance at the ZGS (1), Saturne (2), KEK (3), and until recently at the AGS (4). However, correcting individual resonances becomes impractical for energies above $20 \mathrm{GeV}$.

A full Siberian snake has been proposed as way of overcoming all depolarizing resonances (5), a proposition which has been supported by experimental tests at the IUCF Cooler Ring (6-9). By rotating the proton's spin by $180^{\circ}$ every turn, a full Siberian snake causes the cancellation of depolarizing precessions due to magnetic field errors on alternate rotations through the accelerator. Attractive designs for full Siberian snakes exist for low and high energy accelerators, but no simple solutions exist for medium energy accelerators, like the Fermilab $8 \mathrm{GeV}$ Booster (10) or the Brookhaven AGS (11). In such accelerators partial Siberian snakes might be used to overcome many of the depolarizing resonances.

Without a Siberian snake, each proton's spin precesses around the ring's vertical magnetic field, which is the stable spin direction in this case. However, horizontal magnetic field errors may depolarize the beam when the spin 
precession tune $\nu_{\varepsilon}$, which is the number of times the spin precesses around the stable spin direction per turn in the accelerator, satisfies the following condition

$$
\nu_{s}=n+m \nu_{y},
$$

where the vertical betatron tune $\nu_{y}$ is the number of vertical betatron oscillations per turn in the accelerator, and $n$ and $m$ are integers. Imperfection depolarizing resonances occur when $m=0$, while first-order intrinsic resonances occur when $m= \pm 1$. Without a Siberian snake the spin tune is given by $\nu_{s}=G \gamma$ where $\gamma$ is the Lorentz energy factor and $G=1.792847$ is the proton's anomalous magnetic moment.

A recent experiment (8) confirmed that, in a ring containing a partial Siberian snake of strength $s$, the spin tune is found from

$$
\cos \left(\pi \nu_{s}\right)=\cos (\pi G \gamma) \cos \left(\frac{\pi s}{2}\right),
$$

where $s=1$ corresponds to a full snake, for which $\nu_{s}$ is a half-integer and independent of the energy. On the other hand, for a weak partial Siberian snake, the spin tune is changed from $G \gamma$ but only strongly in the vicinity of the imperfection resonances. Thus, partial Siberian snakes may be useful for overcoming the many weak imperfection resonances in medium energy accelerators, while the comparatively fewer intrinsic depolarizing resonances might be overcome using pulsed quadrupoles to jump the vertical betatron tune as the resonances are approached.

We report here the results of the first acceleration of a polarized proton beam through a depolarizing resonance using a partial Siberian snake, much of which have been recently published elsewhere (12). Not long after our work, a partial Siberian snake was independently tested at the AGS (13). It should also be noted that partial Siberian snakes have been previously used to overcome an imperfection resonance for accelerated polarized positrons (14).

\section{EXPERIMENTAL METHODS AND RESULTS}

The snake strength $s$ of a single solenoid magnet with $N I$ ampere-turns is given by

$$
s=\frac{\mu_{0}(1+G)}{10.479 p} N I,
$$

where $\mu_{0}=4 \pi \times 10^{-7} \mathrm{~T} \mathrm{~m} \mathrm{~A} \mathrm{~m}^{-1}$ and $p$ is the proton's momentum in $\mathrm{GeV} / \mathrm{c}$. Thus to maintain a constant snake strength as the beam is accelerated, the solenoid current must be ramped. For our studies, we installed two rampable warm solenoid magnets symmetrically around our previously installed superconducting solenoid in the IUCF Cooler Ring, separated only by drift spaces. The superconducting solenoid, the polarimeter and the Cooler Ring's operation with polarized protons were discussed earlier $(6-9,15-17)$. The two warm solendoids' currents were varied together so that, in combination with the superconducting solenoid operating at constant current, a constant snake 


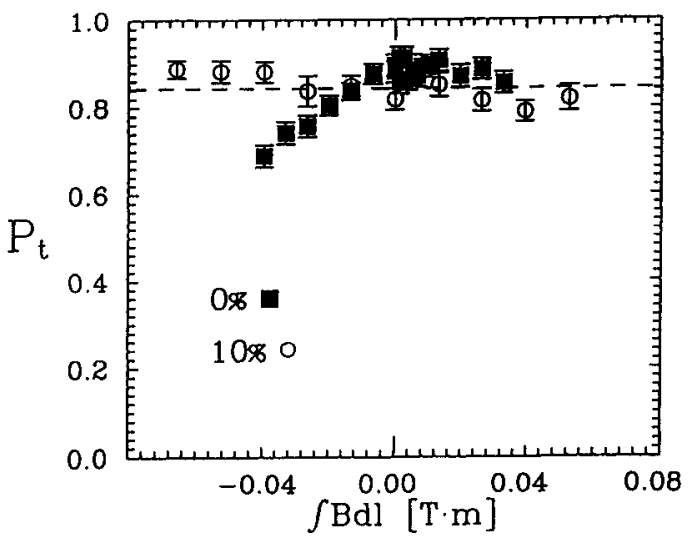

FIGURE 1. The measured transverse polarization $P_{t}$ after acceleration to $140 \mathrm{MeV}$ plotted against the imperfection $\int B d \ell$ with no snake and with a constant $10 \%$ partial Siberian snake. The dashed line is the best constant polarisation fit to the snake-on data.

strength was maintained during acceleration (12). This combination of magnets allowed constant partial Siberian snake strengths of $10 \%, 20 \%$, and $30 \%$ while accelerating from $95 \mathrm{MeV}$ to $140 \mathrm{MeV}$. This energy range includes the $G \gamma=2$ imperfection depolarizing resonance (6) near $108 \mathrm{MeV}$, but is well below $177 \mathrm{MeV}$ where the $7-\nu_{y}$ intrinsic depolarizing resonance normally occurs (7).

We first studied the depolarization during the acceleration with no Siberian snake $(0 \%)$, and then with a $10 \%$ partial Siberian snake. In each study the beam polarization transverse to the beam motion, $P_{t}$, was measured after accelerating to $140 \mathrm{MeV}$ for different values of the imperfection $\int B d \ell$, which is produced by the correction solenoid magnets in the ring's cooling section. The results of these measurements are shown in Fig. 1. It should be noted that the spin direction was actually flipped both with and without the partial Siberian snake, and there is a normalization uncertainity of about $15 \%$ for the values of $P_{t}$, which does not affect the shape of the curves.

With a $0 \%$ snake, there is a significant decrease in $P_{t}$ for nonzero $\int B d \ell$ due to the $G \gamma=2$ imperfection depolarizing resonance. Since the beam accelerated through the resonance with $\langle d \gamma / d t\rangle=0.061 \mathrm{~s}^{-1}$, this $P_{t}$ curve is flatter than the $104 \mathrm{MeV}$ fixed-energy data of Ref. (6); nevertheless, with no snake, the $G \gamma=2$ resonance clearly depolarized the accelerated beam, or rotated its spin into the unmeasurable longitudinal direction. However, with a $10 \%$ partial Siberian snake, the beam polarization measured after acceleration to $140 \mathrm{MeV}$ was almost independent of the imperfection $\int B d \ell$ within our precision of about $2 \%$.

We also studied the effect of stronger partial Siberian snakes. In Fig. 2, the transverse beam polarization, measured after acceleration from 95 to $140 \mathrm{MeV}$, is plotted against the imperfection $\int B d \ell$ for constant partial Siberian snake strengths of $0 \%, 10 \%, 20 \%$, and $30 \%$. Note the behavior in the $20 \%$ and $30 \%$ snake data; the polarization was unexpectedly lost for higher $\int B d \ell$. A possible explanation for this depolarization may be deduced from Fig. 3, in which the calculated values of the spin tune are plotted versus $G \gamma$ for various snake strengths. The spin tunes at which the imperfection and intrinsic resonances occur are marked with horizontal dashed lines, and the values of $\boldsymbol{G} \gamma$ for the 


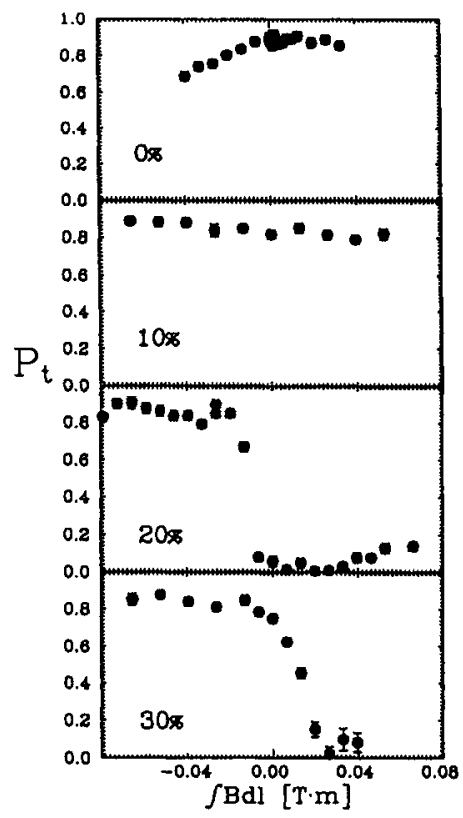

FIGURE 2. The measured transverse polarisation at $140 \mathrm{MeV}$ plotted against the imperfection $\int B d \ell$ for a partial Siberian snake with strengths $0 \%, 10 \%, 20 \%$ and $30 \%$.

initial ( $95 \mathrm{MeV}$ ) and final ( $140 \mathrm{MeV}$ ) energies during the ramp are marked by vertical dashed lines. As long as the spin tune (solid lines) do not pass through a resonance within the range of $G \gamma$ during the ramp, taking into account that the resonance has some width, depolarization should not be observed. Without a Siberian snake, $\nu_{s}=G \gamma$, and the intrinsic resonance would not be encountered until the proton kinetic energy reaches $177 \mathrm{MeV}$. As can be seen in Fig. 3, Siberian snake strengths between $20 \%$ and $30 \%$ result in spin tunes relatively near to the intrinsic depolarizing resonance within our energy range. Although this suggests how the beam may have been depolarized, we do not yet have a detailed explanation for actual depolarization we observed.

To summarize, the $10 \%$ snake data support the conjecture that a weak partial Siberian snake can maintain full beam polarization during acceleration through a weak imperfection depolarizing resonance. Therefore, partial Siberian snakes might be useful for accelerating a polarized proton beam at medium energy accelerators such as the Fermilab $8 \mathrm{GeV}$ Booster (10) or the Brookhaven AGS (11). However, the effect of partial Siberian snakes near intrinsic depolarizing resonances requires further study. Experimental studies are underway which show promise for explaining the depolarization observed with partial snakes with strengths greater than $10 \%$.

We would like to thank J. M. Cameron and the entire Indiana University Cyclotron Facility staff for the most successful operation of the Cooler Ring. We are grateful to V. A. Anferov, A. W. Chao, S. V. Gladysheva, R. S. Herdman, S. Hiramatsu, F. Z. Khiari, A. V. Koulsha, W. F. Lehrer, H.-O. Meyer, J. B. Muldavin, R. E. Pollock, T. Roser, H. Sato, D. S. Shoumkin, S. E. Sund, 


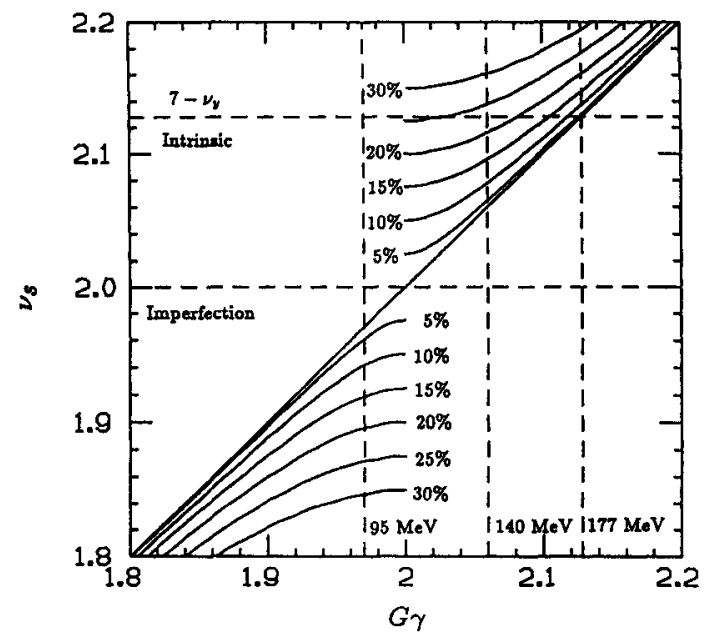

FIGURE 3. Spin tune $\nu_{0}$ versus $G \gamma$ (solid lines) for various partial Siberian snake strengths, as calculated from Eq. 2.

T. Toyama, and S. J. Wheeler for their help with parts of this experiment. This research was supported by grants from the U.S. Department of Energy and the U.S. National Science Foundation.

\section{REFERENCES}

(a) Also at Moscow State University, Moscow, Russia.

(b) Also at Brookhaven National Laboratory, Brookhaven, NY 11973.

(c) Also at Department of Nuclear Engineering, University of Michigan.

(d) Now at Stanford Linear Accelerator Center, Stanford, CA 94309.

(e) Also at Office of the Provost, University of Michigan at Flint, Flint, MI 48501.

1. T. Khoe et al., Part. Accel. 6, 213 (1975).

2. J. L. Laclare et al., J. Phys. (Paris), Colloq. 46, C2-499 (1985).

3. H. Sato et al., Nucl. Instrum. Methods Phys. Res. Sect. A 272, 617 (1988).

4. F. Z. Khiari et al., Phys. Rev, Ds9, 45 (1989).

5. Ya.S. Derbener and A. M. Kondratenko, Part. Accel. 8, 115 (1978).

6. A. D. Krisch et al., Phys. Rev. Lett. 63, 1137 (1989).

7. J. E. Goodwin et al., Phys. Rev. Lett. 64, 2779 (1990).

8. V. A. Anferov et al., Phys. Rev. A46, R7383 (1992).

9. R. Baiod et al., Phys. Rev. Lett. 70, 2557 (1993).

10. Acceleration of Polarized Protons to 120 and $150 \mathrm{GeV}$ in the Fermilab Main Injector, SPIN Collaboration, Univ. of Michigan Report (March 1992), (unpublished).

11. RHIC Spin Proposal M. Beddo et al.; Brookhaven National Lab proposal (1992), (unpublished).

12. B.B. Blinov et al., Phys. Rev. Lett. 73, 1621 (1994).

13. H. Huang et al., these proceedings.

14. Ya.S. Derbenev et al., Proc. of the $X$ International Conference on High Energy Accelerators, Protvino, 1977.

15. R.A. Phelps et al., Phys. Rev. Lett. 72, 1479 (1994).

16. M.G. Minty et al., Phys. Rev. D44, R1361 (1991).

17. J.E. Goodwin, Ph.D. Thesis, Indiana University (1990). 